\title{
Are current avian influenza vaccines a solution for
}

\section{smallholder poultry farmers? [version 1; peer review: 1}

\section{approved, 3 approved with reservations]}

\author{
Vincent Guyonnet ${ }^{1}$, Andew R. Peters (1D2 \\ ${ }^{1}$ FFI Consulting Ltd., Brockville, Ontario, Canada \\ ${ }^{2}$ Supporting Evidence Based Interventions (SEBI), University of Edinburgh, Edinburgh, UK
}

\author{
V1 First published: 26 Aug 2020, 4:122 \\ https://doi.org/10.12688/gatesopenres.13171.1 \\ Latest published: 26 Aug 2020, 4:122 \\ https://doi.org/10.12688/gatesopenres.13171.1
}

\begin{abstract}
Vaccination against highly pathogenic avian influenza (HPAI) viruses, along with other measures, was successful in eradicating AI in very few countries where the competence of national veterinary services or the geography and bird density have contributed favorably to the outcome. The main constraints to an effective AI vaccination are vaccine composition matching field strains, reliable cold chain and logistics to target all poultry smallholders, constraints related to the availability of sufficient financial and human resources. When not conducted properly, vaccination can also contribute to the emergence of new field viral strains, through genetic drifts of HPAI viruses. While new technologies have improved the possibility to produce high quality vaccines matching field strains, recurrent issues like postvaccination field surveillance and vaccination coverage continue to limit the relevance of AI vaccination in smallholder settings. A "gamechanger" vaccine targeting smallholders should be universal to protect against all field viral strains and reduce significantly, if not totally eliminate, the need for costly post-vaccination surveillance. The ease of administration of this vaccine (eye drop or one single injection) would further contribute to its relevance in the field. These characteristics are considered essential for the product profile of an AI vaccine that can contribute in a meaningful way to the livelihoods of poultry smallholders.
\end{abstract}

Keywords

Avian Influenza, vaccines, vaccination, poultry smallholders

\section{Open Peer Review}

$\begin{array}{ccccc}\text { Approval Status } & ? ? & ? \\ & 1 & 2 & 3 & 4 \\ \text { version 1 } & ? & ? & \checkmark & ? \\ 26 \text { Aug 2020 } & \text { view } & \text { view } & \text { view } & \text { view }\end{array}$

1. Luca Busani ID, Istituto Superiore di Sanità, Rome, Italy

2. Gavin J.D. Smith ID, Duke-NUS Medical

School, Singapore, Singapore

3. Erica Spackman ID, United States

Department of Agriculture (USDA)-

Agricultural Research Service (ARS), Athens,

USA

4. Leslie D. Sims (iD), Asia Pacific Veterinary Information Services Pty. Ltd, Montmorency, Australia

Any reports and responses or comments on the article can be found at the end of the article.

This article is included in the AgriKnowledge

$$
\text { or AgriKnowledge gateway. }
$$


Corresponding author: Andew R. Peters (andy.peters@ed.ac.uk)

Author roles: Guyonnet V: Data Curation, Formal Analysis, Investigation, Methodology, Writing - Original Draft Preparation; Peters AR: Conceptualization, Funding Acquisition, Methodology, Project Administration, Writing - Review \& Editing

Competing interests: No competing interests were disclosed.

Grant information: This work was supported by the Bill and Melinda Gates Foundation [INV-009921].

The funders had no role in study design, data collection and analysis, decision to publish, or preparation of the manuscript.

Copyright: (c) 2020 Guyonnet V and Peters AR. This is an open access article distributed under the terms of the Creative Commons Attribution License, which permits unrestricted use, distribution, and reproduction in any medium, provided the original work is properly cited.

How to cite this article: Guyonnet $V$ and Peters AR. Are current avian influenza vaccines a solution for smallholder poultry farmers? [version 1; peer review: 1 approved, 3 approved with reservations] Gates Open Research 2020, 4:122

https://doi.org/10.12688/gatesopenres.13171.1

First published: 26 Aug 2020, 4:122 https://doi.org/10.12688/gatesopenres.13171.1 


\section{Introduction}

Although more than 30 epizootics of high pathogenicity Avian Influenza (HPAI) have been reported in poultry (Gallus domesticus) and other birds since 1959, vaccination of Poultry has only been added as a control tool since 1995 (Swayne et al., 2014). In recent outbreaks, vaccination has been used only in about $19 \%$ of the countries experiencing HPAI 15 countries out of 80 countries), showing that vaccination is not the most common and immediate response to an outbreak (Swayne et al., 2011). The nature of the AI virus, with the rapid emergence of new field viral strains, through genetic drifts of HPAI viruses, has affected the effectiveness of vaccines. Vaccination along with other measures has been successful in eradicating AI in very few countries where the competence of national veterinary services (e.g. France, the Netherlands) or the geography and bird density (Hong Kong) have favorably contributed to the outcome. In the four countries (China, Egypt, Indonesia and Vietnam) where massive vaccination campaigns have been initiated since 2004 (accounting for more than $99 \%$ of the use of AI vaccines), HPAI virus is now endemic, outlining the difficulty to eradicate AI in countries with a high percentage of backyard poultry (Swayne et al., 2011). A number of factors are limiting the effectiveness of AI vaccination in smallholder settings and are discussed in the following sections along with the characteristics of the ideal vaccine to target the poultry smallholder segment.

\section{AI - etiology and epidemiology}

Influenza viruses belong to the Orthomyxoviridae family, causing respiratory disease of the upper respiratory tract in humans, avian species, and a variety of mammal species. Orthomyxoviruses are classified as Types A, B or C with Avian influenza caused by a highly mutable Type A influenza virus.

The replication of this single stranded RNA virus is highly variable, resulting in a constantly evolving and highly mutable virus (Swayne et al., 2013). The lipid envelope of the virus makes it unstable and relatively susceptible to environmental destruction from ultraviolet light, chemicals, or desiccation. The hemagglutinin $(\mathrm{HA}$ or $\mathrm{H})$ antigen is a protein providing the mechanism for the entry into the host cell while the neuraminidase (NA or $\mathrm{N})$ protein allows the exit of newly replicated virions from the host cells. The HA protein is the major antigen stimulating the host immune response with protective antibodies against clinical signs and mortality. There are at least $16 \mathrm{H}$ strains and $9 \mathrm{~N}$ strains, which can result in 144 possible HN combinations. This forms the basis of the serological classification using the hemagglutinin inhibition and neuraminidase inhibition tests. The nomenclature of the virus is based on the HN subtype, influenza type, host species, sample location, strain number and year of isolation, e.g. H5N1 A/goose/Guangdong/1/1996.

The antigenic variation of the HA and NA surface glycoproteins occurs at a high frequency through minor "drift" changes and may be associated with the immune pressure exerted by the vaccination of birds. Major antigenic "shift" in the HA and NA coding proteins, the result of genetic re-assortment between gene segments of two different influenza virus strains (subtypes) in host cells, commonly occurs especially when domestic waterfowl and poultry are in close proximity as often seen in developing countries under smallholder settings. Ducks are often silent carriers of the AI virus, constituting an additional challenge for the control of AI (Swayne et al., 2013). AI viruses in poultry are classified as either low pathogenic (LPAI) or highly pathogenic (HPAI), based on the clinical signs and mortality, using the definition established by the World Organization for Animal Health (OIE, 2018). During the period January 2013 to February 2019, HPAI in domestic poultry was reported in 68 countries and territories with a total of 7,270 outbreaks (OIE, 2019). In most of the countries in Asia and Africa, the poultry sector is dominated by smallholder farmers.

During the same period, a total of 12 subtypes of AI viruses were reported (OIE, 2019), with subtypes H5N1, H5N2 and H5N8 widespread and more frequently reported (Table 1).

LPAI outbreaks with viruses of $\mathrm{H} 5$ and $\mathrm{H} 7$ subtypes are also reportable to the OIE as there is a risk of the viruses becoming highly pathogenic by mutation. During the period January 2013-February 2019, the vast majority of these outbreaks were reported in Asia, Europe, the Americas and a few in Africa (mainly in South Africa).

H5N1 HPAI viruses have been sub-classified according to Clades, or groups of AI viruses that share a common ancestor (WHO, 2011). There are at least 10 Clade groups currently identified by the OIE and FAO network of expertise on animal influenza (OFFLU). Both antigenic shift and drift are important mechanisms for the evolution of the virus. The presence of co-circulating subtypes among dense populations of birds adds extra pressure for antigenic shift. Intrinsic subtype specific antigenic drift is associated with the frequency and distribution of infection in a poultry population as naive populations become exposed to new variants. Vaccination is also believed to exert a selection pressure on the virus by increasing the mutation rate by several orders of magnitude (Swayne et al., 2014). In addition, the evolution of new clade types can change the morbidity and mortality. Prior to 2012, clade 2.2 (Indonesia) and clade 1.1 (Cambodia) were predominant, with mortality in domestic ducks of less than $10 \%$. Following the introduction of clade 3.2.1, the mortality reported in ducks is greater than $40 \%$ and up to $90 \%$ depending on the age of

Table 1. Distribution of different HPAI subtypes in domestic birds, by region (January 2013 - February 2019).

\begin{tabular}{|l|l|}
\hline Region & HPAI subtypes \\
\hline Africa & $\mathrm{H} 5 \mathrm{~N} 1, \mathrm{H} 5 \mathrm{~N} 2, \mathrm{H} 5 \mathrm{~N} 8$ \\
\hline Americas & $\mathrm{H} 5 \mathrm{~N} 1, \mathrm{H} 5 \mathrm{~N} 2, \mathrm{H} 5 \mathrm{~N} 8, \mathrm{H} 7 \mathrm{~N} 3, \mathrm{H} 7 \mathrm{~N} 8, \mathrm{H} 7 \mathrm{~N} 9$ \\
\hline Asia & $\mathrm{H} 5 \mathrm{~N} 1, \mathrm{H} 5 \mathrm{~N} 2, \mathrm{H} 5 \mathrm{~N} 3, \mathrm{H} 5 \mathrm{~N} 6, \mathrm{H} 5 \mathrm{~N} 8, \mathrm{H} 7 \mathrm{~N} 9$ \\
\hline Europe & $\mathrm{H} 5 \mathrm{~N} 1, \mathrm{H} 5 \mathrm{~N} 2, \mathrm{H} 5 \mathrm{~N} 5, \mathrm{H} 5 \mathrm{~N} 6, \mathrm{H} 5 \mathrm{~N} 8, \mathrm{H} 5 \mathrm{~N} 9, \mathrm{H} 7 \mathrm{~N} 7$ \\
\hline Oceania & $\mathrm{H} 7 \mathrm{~N} 2$ \\
\hline
\end{tabular}


the ducks. As a virus is transferred from wild birds to poultry, natural selection within the host favours greatly adapted strains, which often become more pathogenic for both LPAI and HPAI pathotypes (Swayne et al., 2013).

The type of poultry production is also critical to assess the risk of exposure. Birds kept for longer periods (laying hens, breeder birds and slow-growth meat birds) have a longer duration of potential exposure to AI virus than short-lived poultry (broiler-type meat birds). The concurrent presence of immunosuppressive agents and conditions for example, reduce the infectious dose and has been associated with morbidity and mortality due to both LPAI and HPAI infections. Therefore, general health screening for concurrent diseases in poultry, often difficult to achieve under smallholder settings, is important during immunization and for the overall control of avian influenza.

AI outbreaks during the period January 2013-February 2019 resulted in the loss of approximately 128 million birds, with more than half $(57.6 \%)$ of the reported losses in Asia, followed by the Americas (22.1\%) and Europe (13.4\%) (OIE, 2019). Losses in Africa accounted for only $6.5 \%$ of the total losses (Table 2).

\section{AI vaccines and vaccination}

AI vaccine types and production

Poultry AI vaccines used in the field are based on five technologies: 1) wild-type or reverse genetics whole AI virus grown in embryonated chicken eggs, then chemically inactivated and adjuvanted; 2) HA antigen or virus-like particles produced in insect cells by a genetically engineered baculovirus; 3) HA DNA vaccine adjuvanted; 4) recombinant technologies utilizing live virus vectors to express AI virus HA and in some cases NA gene inserts (recombinant Herpes turkey virus (rHVTAIV), recombinant Newcastle disease virus (rNDV-AIV) and recombinant Fowl pox virus (rFPV-AIV)) and, 5) defectivereplicating alphavirus (defective Venezuelan Equine Encephalitis virus with H5 AI virus gene insert (D. E. Swayne, personal communication).

The recombinant ND-vectored vaccines used for the control of AI are broadly divided into two categories (Suarez et al., 2017).

\section{Table 2. Distribution of mortality in domestic birds, by region (January 2013 - February 2019).}

\begin{tabular}{|l|l|c|}
\hline Region & Losses & $\begin{array}{c}\text { Percentage } \\
\text { of total }\end{array}$ \\
\hline Africa & $8,339,384$ & $6.5 \%$ \\
\hline Americas & $28,224,324$ & $22.1 \%$ \\
\hline Asia & $73,673,631$ & $57.6 \%$ \\
\hline Europe & $17,187,364$ & $13.4 \%$ \\
\hline Oceania & 490,000 & $0.4 \%$ \\
\hline Total & $127,914,703$ & $100 \%$ \\
\hline
\end{tabular}

One alternative uses reverse genetic technology to insert an AI gene sequence coding for a specific protein into the ND virus thus producing a recombinant ND virus expressing that protein. After viral multiplication in embryonated chicken eggs, the recombinant viruses recovered are inactivated and adjuvanted for the production of the AI vaccine. This technology was recently patented in the USA by Laboratorio Avimex (Lozano-Dubernard et al., 2015) and presents the advantage of being safe to use in BSL-2 production facilities, more readily available in developing countries.

The second alternative applies the reverse technology to produce live ND vector vaccine viruses, often using the more aggressive LaSota vaccinal strain as the backbone, which express HA genes (H5, H6, H7 and $\mathrm{H} 9$ inserts). One of the primary benefits of this alternative is that the live virus replicates on mucosal surfaces, can be administered by mass application such as water or aerosol application, thus reducing the overall cost of vaccination and may be administered to other species of poultry, especially ducks. Conversely, one of the main concerns is related to the level of maternal ND antibodies in chicks which may interfere with the replication of the recombinant ND viruses and overall efficacy of the vaccine. In addition to being considered as genetically modified organisms, the potential of live ND vectored vaccines to spread to non-target species and to unvaccinated flocks often raises concerns during the regulatory review and vaccine licensing process (Suarez et al., 2017).

There are several new experimental AI vaccine approaches not currently licensed for commercial use including wild type or attenuated LPAI; use of various vectors e.g. adenovirus, salmonella, avian leukosis and vaccinia; eukaryotic systems e.g. plants; and DNA vaccines, with a main objective being a universal vaccine covering against all subtypes of HPAI. Of course, this would be an ideal scenario. However current opinion is that there is little expectation that such vaccine will be developed to licensure in the foreseeable future.

\section{Multivalent vaccines commercially available}

Review of the vaccine database from The Center for Food Security and Public Health, Iowa State University showed that of 43 manufacturers listing vaccines for the control of Avian Influenza, $31(72 \%)$ are currently manufacturing vaccines combining AI with other antigens (CFSPH, 2018). These manufacturers are based in 11 different countries on five continents but the majority of them $(71 \%)$ are located in Asia (22 of the 31) where AI vaccination has been practiced since 1995. Manufacturers located in Europe and North America are producing vaccines mostly for export and for the setting-up of emergency stocks. There is currently just one manufacturer of AI vaccines on the African continent, although it is believed that others are at a planning stage.

The combination vaccines produced are either AI + ND only (26 manufacturers, 84\%), AI +ND + other antigens (15 manufacturers, $48 \%$ ) and $\mathrm{AI}+$ another antigen but not ND (3 manufacturers, $10 \%$ ), these numbers accounting for the fact that some manufacturers are producing more than one type of AI combination vaccine. 
Use of AI combination vaccines by the poultry sector

Based on the list of antigens combined with AI presented previously, it is evident that these vaccines are mostly used in the egg laying and breeder poultry sectors and not by poultry smallholders. As might be expected from this wide range of vaccines and the disease challenges experienced in the field, there are a multitude of vaccination schedules used by poultry producers.

As an example, a recent study in Indonesia by Tarigan et al. (2018) showed that there were broadly three types of schedule for the administration of AI vaccines to commercial laying birds:

- two or three vaccinations before 19 weeks of age (start of laying period)

- two vaccinations before 19 weeks of age and one vaccination after 19 weeks

- three or four vaccinations before 19 weeks and 2 or 3 vaccinations after 19 weeks

Irrespective of the vaccination schedule or the specific vaccines used, Tarigan et al. (2018) demonstrated that the birds were not protected throughout production. Without vaccination after 19 weeks, the birds failed to be protected after 38 weeks of age; with vaccination after 19 weeks, the birds failed to be protected around 58 weeks of age. The data clearly demonstrated showed that with laying or breeder birds the duration of immunity is one of the main limitation issues for life cycle protection in the field, especially in the smallholder setting.

Among the recombinant viral-vectored vaccines, the rHVTAIV has the advantage that it can be used in day-old chicks at the hatchery. This vaccine has shown some good results in field studies in Egypt and is currently licensed in five countries (Bangladesh, Egypt, Mexico, Vietnam and the USA (von Dubschuetz, 2013).

\section{Need for regular AI vaccine reformulation}

Due to the antigenic drift of the AI virus in the field, there is a need to constantly update the composition of AI vaccines. This can be achieved only through constant monitoring of the virus strains in the field, rapid regulatory review and approval process and, good manufacturing processes. As the largest user of AI vaccines, the evolution over time of the vaccinal strains used in China is quite indicative of the need for regular reformulation, both for killed and live vaccines (Table 3).

Over the course of 10 years, the control of H5N1 in China has required the introduction of seven different killed vaccines. In addition, H5N1 has also been combined with H9N2 for better vaccination coverage, adding two new killed vaccine formulations (Fan et al., 2015). In 2017, a vaccine combining H7N9 with H5N1 was approved for use in poultry (Shi et al., 2018). The need for the regular updating of $\mathrm{H} 5 \mathrm{~N} 1$ viral subtypes is also required for live recombinant NDV-vectored vaccines, with three different formulations (rLH5-1, rLH5-5 and rLH5-6) approved since 2005 (Fan et al., 2015). The need for regular updating of vaccinal strains was also reported by Tarigan et al. (2018) in Indonesia, another country using vaccination to control avian influenza on a wide scale since the first outbreak was reported in 2003 (Table 4).

Due to the antigenic drift of the AI virus in the field, the geographical coverage of AI vaccines is very specific and sometimes limited to certain provinces or districts in a country. Therefore, most AI vaccines administered in China, Pakistan, Indonesia and Egypt are produced by local manufacturers.

\section{Key learnings from national AI vaccination campaigns}

Since 2002 with the use of vaccines to control H5 HPAI in Hong Kong, mass AI vaccination campaigns have been implemented in China, Vietnam, Indonesia, Egypt and Mexico. Key learnings were shared by these countries during the OFFLU technical meeting in Beijing, China and reported in the recommendations issued (OFFLU, 2013). A number of authors have also reviewed the performance of vaccination campaigns in Egypt (Kaoud, 2017), Indonesia (Swayne et al., 2015), Asia (Peyre et al., 2009) and globally (Pavade et al., 2011) as well as the economic cost and benefits (Hinrichs \& Otte, 2012; Hinrichs, 2013; Sun et al., 2017).

The key learnings from these national AI vaccination campaigns are summarized below:

- Antigenic drift occurs with all AI viruses and can reduce the effectiveness of vaccination over time. It is essential to use vaccinal strains with sufficient quantities of antigens reasonably well matched with circulating strains of AI virus.

\section{Table 3. Evolution of the H5N1 viral strains used in killed vaccines in China (2004-2014).}

\begin{tabular}{|l|l|}
\hline Year & Viral strains in H5N1 vaccines used in China \\
\hline 2004 & Heterologous H5N2 strain, killed \\
\hline 2005 & H5N1 Re-1, killed \\
\hline $2006-2012$ & $\begin{array}{l}\text { Killed H5N1 Re-4, Re-5, Re-6 and bivalent } \\
\text { vaccines killed H5N1 Re-1/Re-4, Re-4/Re-5 and } \\
\text { Re-4/Re-6 }\end{array}$ \\
\hline 2014 & H5N1 Re-7, killed \\
\hline
\end{tabular}

Table 4. Evolution of the H5N1 viral strains used in killed vaccines in Indonesia (2004-2012).

\begin{tabular}{|l|l|}
\hline Year & $\begin{array}{l}\text { Viral strains in H5N1 } \\
\text { vaccines used in Indonesia }\end{array}$ \\
\hline 2004 & H5N1 subclade 2.1.1 \\
\hline 2008 & H5N1 subclade 2.1.2 and 2.1.3 \\
\hline 2012 & H5N1 subclade 2.3.2.1 \\
\hline
\end{tabular}


- Vaccination with high-quality, registered vaccines, according to established protocols reduces resistance to infection, decreases viral shedding and decreases the probability of infection to poultry, other animals and humans.

- Vaccination only partially reduces viral shedding and can promote, especially if not conducted correctly, depending on the choice of vaccine, mode and frequency of administration, the selection of mutation in the circulating virus.

- Vaccination alone cannot eliminate the virus and is only meant to be part of an integrated, holistic control program adapted to local conditions

- It is difficult to maintain high level flock immunity in some poultry populations, especially at small production and backyard levels.

- Vaccination is logistically demanding, and additional costs will be initially incurred in countries lacking efficient cold chain distribution networks required for most vaccines.

- Vaccination is expensive due to the need to conduct high quality post-vaccination surveillance to monitor the genetic and antigenic characteristics of circulating field viruses.

- The return on investment of vaccination must be carefully considered before adding vaccination as part of an AI control programme.

In Indonesia, a survey of commercial egg producers (Brum, 2013) showed that AI vaccinations were ineffective for the following reasons: poor selection of vaccinal strains vs. field challenge, first AI vaccine given too late and, not enough booster vaccinations, especially during the period of egg production (when vaccination will cause a reduction in egg production). Farmers also lacked independent high-quality technical support and $55 \%$ of the respondents said that they needed better information on vaccine selection and the vaccination schedule.

Regarding the situation in countries with large backyard poultry production, Alders et al. (2007); Gardner \& Alders (2014); Pavade et al. (2011) and Sims et al. (2016) have provided some additional points to consider:

- Backyard poultry are extremely hard to reach, and the exercise remains extremely costly. Thus, vaccination should not be started if adequate funding for the preparation, implementation and monitoring cannot be guaranteed.

- As a rule, it is not recommended to vaccinate backyard poultry since maintaining adequate levels of immunity is extremely difficult.

- Community vaccinators are often reluctant to vaccinate backyard birds under 2 months of age for fear of killing them, thereby missing about $50 \%$ of the birds at risk.
- Vaccination is not a substitute for other important measures like biosecurity and can give farmers a false sense of security.

- Poor vaccination practices, insufficient vaccination coverage and lack of post-vaccination monitoring (as often seen in developing countries with large backyard poultry populations) can contribute to an endemic situation.

\section{Overview on government AI control}

Information on national regulations and policies related to $\mathrm{AI}$ vaccination is available via the World Animal Health Information System (WAHIS) interface hosted by the OIE (OIE, 2019). This database is built from information submitted to the OIE by its 184 country members. Among the parameters recorded, the following information is relevant to this paper: vaccination prohibited and official vaccination. It must be noted that official vaccination is not necessarily the converse of vaccination prohibited. Vaccination against poultry diseases may be the result of official vaccination and / or voluntary vaccination.

According to the OIE website (OIE, 2019), the countries with official AI vaccination programs are Mexico, Egypt, Pakistan, Kazakhstan and Russia. China also has official AI vaccination. Vaccination is also allowed in Bangladesh where import permits are delivered to the private sector and killed AI vaccines imported (no combination vaccines used). Vietnam, Egypt and Indonesia have transitioned from mass vaccination to targeted vaccination (OFFLU, 2013). The cost of AI vaccination is often shared between governments and the private sector. For instance, in Vietnam, farmers with $>2,000$ birds pay the full cost of vaccination while farmers with $<2,000$ birds receive some provincial subsidies.

Regional organizations like the African Union and the Association of South-East Asian Nations do not have specific policies regarding AI except for the desire to strengthen the linkages within countries and across borders, for the sharing of information and knowledge and for the development of partnership between all stakeholders in public and private sectors and civil society.

At the intergovernmental level, the Network of Expertise on Animal Influenza (OFFLU), a joint OIE-FAO technical committee with a worldwide network of contributors, issued in 2013 a series of recommendations on vaccination and vaccines (OFFLU, 2013).

\section{The ideal AI vaccine candidate for poultry smallholders}

The desired attributes (product profile) for AI vaccines targeting the smallholder segment are presented in Table 5.

Based on the views of a number of experts from academia, governments, intergovernmental organizations and the private sector, the top desired attributes for the smallholder segment are listed in Table 6. 
Table 5. Desired attributes of AI vaccines addressing the smallholder segment.

\begin{tabular}{|c|c|}
\hline Desired attribute & Current situation \\
\hline Inexpensive & $\begin{array}{l}\text { Current cost for inactivated AIV vaccine: } \$ 0.03-0.10 / \text { dose plus cost of administration } \\
\text { (\$0.05-0.07 per dose for individual handling and injection) }\end{array}$ \\
\hline $\begin{array}{l}\text { Use in multiple avian } \\
\text { species }\end{array}$ & $\begin{array}{l}\text { Most vaccines are used in meat, layer and breeder chickens although a large number of } \\
\text { doses also used in ducks; minor amounts in turkeys, geese, quail, etc. }\end{array}$ \\
\hline Single dose protection & $\begin{array}{l}\text { Most situations require a minimum of } 2 \text { doses; prime-boost scenario is optimal with } \\
\text { revaccination in long-lived birds at 6-12-month intervals }\end{array}$ \\
\hline Mass application & $\begin{array}{l}95.5 \% \text { is inactivated vaccine administered by handling and injecting individual birds and } 4.5 \% \\
\text { as vectored vaccine given by mass spray vaccination }\end{array}$ \\
\hline $\begin{array}{l}\text { Identify infected birds in } \\
\text { vaccinated population (DIVA) }\end{array}$ & $\begin{array}{l}\text { Serological differentiation tests are available, but only minor use. Most vaccine applied } \\
\text { without using a serological DIVA strategy for surveillance }\end{array}$ \\
\hline $\begin{array}{l}\text { Overcome maternal } \\
\text { antibody interference }\end{array}$ & $\begin{array}{l}\text { Maternal antibody to AIV hemagglutinin or virus vector inhibits primary immune response. } \\
\text { Initial vaccination must be timed for declining maternal antibody titers to allow optimal } \\
\text { primary immune response }\end{array}$ \\
\hline $\begin{array}{l}\text { Given at } 1 \text { day of age in } \\
\text { hatchery or in ovo }\end{array}$ & $\begin{array}{l}\text { Inactivated vaccine provides poor protection when given at } 1 \text { day of age. Vectored vaccines } \\
\text { can be given at } 1 \text { day of age, but generally require a boost with inactivated vaccine } 10 \text { days } \\
\text { or more later }\end{array}$ \\
\hline Universal vaccine & $\begin{array}{l}\text { The majority of inactivated whole AIV vaccines use reverse genetic generated vaccine seed } \\
\text { strains to antigenically match field viruses. The vaccinal strain of virus should also be a } \\
\text { strong immunogen }\end{array}$ \\
\hline Thermostable & $\begin{array}{l}\text { Killed AI vaccines, rNDV-AI and rFPV-AI vaccines require refrigeration and rHVT-AI vaccine } \\
\text { must be stored in liquid nitrogen }\end{array}$ \\
\hline
\end{tabular}

Table 6. Desired attributes of AI vaccines addressing the smallholder segment.

\begin{tabular}{|l|l|}
\hline $\begin{array}{l}\text { Desired } \\
\text { attribute }\end{array}$ & Rationale \\
\hline $\begin{array}{l}\text { Universal } \\
\text { vaccine }\end{array}$ & $\begin{array}{l}\text { The antigenic drift of the AI viruses in the field requires a constant surveillance usually } \\
\text { poorly performed by national veterinary services in developing countries due to the lack } \\
\text { of proper facilities, human and financial resources. In addition, the availability of universal } \\
\text { vaccines would eliminate the need to regularly update vaccines, often affected by } \\
\text { inefficient national regulatory review and approval processes for vaccines }\end{array}$ \\
\hline $\begin{array}{l}\text { Use in multiple } \\
\text { species }\end{array}$ & $\begin{array}{l}\text { Ducks often act as silent carriers, shedding AI virus without expressing clinical signs. An } \\
\text { effective vaccine in ducks as well as chickens would be of greater value, especially in South } \\
\text { East Asia }\end{array}$ \\
\hline $\begin{array}{l}\text { Single dose } \\
\text { protection }\end{array}$ & $\begin{array}{l}\text { The biggest logistical hurdle and highest cost of AI vaccination is related to the handling } \\
\text { and injection of each bird. A vaccine conferring at least a 6-month protection after one } \\
\text { single administration would maximize the value of mass vaccination campaigns }\end{array}$ \\
\hline
\end{tabular}

Due to the genetic drift of the AI viruses, the current commercial vaccines have a limited geographical coverage and use. The development of a universal vaccine would allow for the same commercial vaccine to be used in all major countries currently vaccinating against AI (China, Egypt, Indonesia, Vietnam and Mexico). Such a vaccine would be also available in countries considering adding vaccination to their AI control measures even though the current trend shows that fewer countries are willing to initiate AI vaccination.

\section{Conclusion}

Vaccination against HPAI has been used as one of the tools in national AI control programmes with varying degrees of successes. Few countries have been able to eradicate HPAI and these countries have typically relied on extremely competent national veterinary services and / or had limited poultry population at risk. Current vaccines, when well-matched to field strains (in quantity and quality), administered properly (timing, frequency and method of administration) and combined 
with a wide range of other measures have prevented financial losses in poultry farms and reduced the risks to human populations.

The cost of AI vaccines is only a small part of the overall cost of vaccinating poultry against AI, especially when targeting poultry smallholders in lower- and middle-income countries. The current vaccine production technologies and vaccines have not been able to address the cost inherent to their use. In order to represent a significant advance, the profile for vaccine candidates must include protection against all viral strains (universal vaccine), efficacy in ducks with reduction in viral shedding and, protection after a single administration. Without the inclusion of these properties in the vaccine profile, it is unlikely that any vaccine candidate would add significant value to the current AI vaccine market and thus warrant the investment of funds for its development.

\section{Data availability}

No data are associated with this article.

\section{Disclaimer}

The views expressed in this article are those of the authors. Publication in Gates Open Research does not imply endorsement by the Gates Foundation.
Alders RG, Bagnol B, Young MP, et al.: Challenges and constraints to vaccination in developing countries. In: Dodet, B. and the Scientific \& Technical Department of the OIE (eds), Vaccination: A tool for the control of Avian Influenza, Dev Biol (Basel). 2007; 130: 73-82.

PubMed Abstract

Brum E: Farmers' perceptions on $\mathrm{H} 5$ vaccination (and other interesting topics). OFFLU Technical meeting, December 4-6, 2013. Beijing China. 2013. Accessed 12 October 2018.

Reference Source

CFSPH: Vaccine database. The Center for Food Security and Public Health Iowa State University, www.cfsph.iastate.edu, 2018. Accessed on October 11, 2018.

Reference Source

Fan X, Hu Y, Zhang G, et al.: Veterinary influenza vaccines against avian influenza in China. Future Virol. 2015; 10(5): 585-595.

Publisher Full Text

Gardner E, Alders R: Livestock risks and opportunities: Newcastle Disease and Avian Influenza in Africa. GRF Davos Plant@Risk. 2014; 2(4): 208-211. Reference Source

Hinrich s: Review on cost and financial implications of HPAI vaccination in countries applying HPAI vaccination. OFFLU Technical meeting, December 4-6, 2013. Beijing China. 2013. Accessed 12 October 2018.

Reference Source

Hinrichs J, Otte J: Large-scale vaccination for the control of Avian Influenza - Epidemiological and Financial implications. In: Zilberman, D., Otte, J., Roland-Holst, D. \& Pfeiffer, D. (Eds), Health and animal agriculture in developing countries, (Springer / FAO). 2012; 207-231.

Publisher Full Text

Kaoud HA: Causes of failure to eradicate avian influenza in Egypt. Open Access / Sci. 2017; 1(4): 104-105.

Publisher Full Text

Lozano-Dubernard B, Sarfati-Mizrahi D, Suarez-Martinez JA, et al.: Recombinant inactivated viral vector vaccine. Patent US 2015/0056245 A1, USPTO http:// patft.uspto.gov/. 2015. Accessed 1 October 2018.

Reference Source

OFFLU: OFFLU technical meeting: Developing guidance on vaccines and vaccination against HPAI from lessons learned. 2013.

Reference Source

OIE: Chapter 10.4 - Infection with avian influenza viruses. Terrestrial Animal Health Code. 2018. Accessed on May 27, 2019.

Reference Source

OIE: World Animal Health Information Database (WAHIS) interface. 2019. Accessed on May 27, 2019.

Reference Source

Pavade G, Awada I, Hamilton K, et al.: The influence of economic indicators, poultry density and the performance of Veterinary Services on the contro of high-pathogenicity avian influenza in poultry. Rev Sci Tech. 2011; 30(3):
661-671.

PubMed Abstract | Publisher Full Text

Peyre M, Fusheng G, Desvaux S, et al.: Avian Influenza vaccines: a practical review in relation to their application in the field with a focus on the Asian experience. Epidemiol Infect. 2009; 137(1): 1-21.

PubMed Abstract | Publisher Full Text

Shi J, Deng G, Ma S, et al.: Rapid Evolution of H7N9 Highly Pathogenic Viruses that Emerged in China in 2017. Cell Host Microbe. 2018: 24(4): 558-568.e7.

PubMed Abstract | Publisher Full Text | Free Full Text

Sims L, Tripodi A, von Dobschuetz S, et al.: Rational use of vaccination for prevention and control of $\mathbf{H 5}$ highly pathogenic Avian Influenza. FAO Focus On May 2016. 2016; 10.

Reference Source

Suarez DL, Pantin-Jackwood MJ: Recombinant viral-vectored vaccines for the control of avian influenza in poultry. Vet Microbiol. 2017; 206: 144-151. PubMed Abstract | Publisher Full Text

Sun Z, Wang J, Huang Z: Assessment of China's H5N1 routine vaccination strategy. Sci Rep. 2017; 7: 46441

PubMed Abstract | Publisher Full Text | Free Full Text

Swayne DE, Pavade G, Hamilton $\mathrm{K}$, et al:: Assessment of national strategies for control of high-pathogenic avian influenza and low-pathogenic notifiable avian influenza in poultry, with emphasis on vaccines and vaccination. Rev Sci Tech. 2011; 30(3): 839-870.

PubMed Abstract | Publisher Full Text

Swayne DE, Spackman E, Pantin-Jackwood M: Success factors for Avian Influenza vaccine use in Poultry and potential impact at the Wild Bird - Agricultural interface. EcoHealth. 2014; 11(1): 94-108.

PubMed Abstract | Publisher Full Text

Swayne DE, Suarez DL, Sims LD: Chapter 6 - Influenza. In: Diseases of Poultry,

$13^{\text {th }}$ edition, D.E. Swayne (Ed), (Wiley \& Sons Inc.). 2013; 181-218.

Publisher Full Text

Swayne DE, Suarez DL, Spackman E, et al.: Antibody titer has positive predictive value for vaccine protection against challenge with natural antigenic-drift variants of H5N1 high-pathogenicity Avian Influenza viruses from Indonesia. J Virol. 2015; 89(7): 3746-3762.

PubMed Abstract | Publisher Full Text | Free Full Text

Tarigan S, Wibowo MH, Indriani R, et al.: Field effectiveness of highly pathogenic avian influenza H5N1 vaccination in commercial layers in Indonesia. PLoS One. 2018; 13(1): e0190947.

PubMed Abstract | Publisher Full Text | Free Full Text

von Dubschuetz S: Implications and implementation of day-old chick vaccination. OFFLU Technical meeting, December 4-6, 2013. Beijing China. 2013. Accessed 12 October 2018

Reference Source

WHO: Updated unified nomenclature for the highly pathogenic H5N1 avian influenza viruses. 2011. Accessed on November 6, 2018.

Reference Source 


\section{Open Peer Review}

\section{Current Peer Review Status:

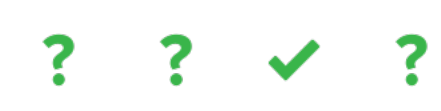

Version 1

Reviewer Report 26 October 2020

https://doi.org/10.21956/gatesopenres.14370.r29532

(C) 2020 Sims L. This is an open access peer review report distributed under the terms of the Creative Commons Attribution License, which permits unrestricted use, distribution, and reproduction in any medium, provided the original work is properly cited.

\section{Leslie D. Sims}

Asia Pacific Veterinary Information Services Pty. Ltd, Montmorency, Vic, Australia

The paper provides an interesting commentary and suggestions on vaccination against highly pathogenic avian influenza. In general, the recommendations are reasonable that better vaccines with the properties described would be valuable. However, there are areas where revision should be considered given the population of farmers targeted has not been fully defined, all reasons for vaccination have not been covered, the authors have not always provided sufficient balance between the problems associated with vaccination and the solutions available, and could have provided more information on new developments in vaccination. In addition, availability of suitable vaccines is not necessarily the main reason for not applying vaccination.

The paper focuses on mass public sector vaccination programmes, in particular vaccination against Gs/GD/96-lineage highly pathogenic H5Nx avian influenza. However, in several countries where these viruses are endemic and vaccination is used against this disease, no formal government programme is in place (the role of government is to approve vaccines for use). It is up to individual farmers to purchase and administer the vaccine (or get someone to do it for them).

As the authors point out, vaccination of small flocks against avian influenza can be difficult due to logistical difficulties, and rapid turnover in multi-age flocks. It has been demonstrated in Egypt and Indonesia that it is not sustainable as a government-sponsored programme. However, there is no technical reason why a well-matched, relatively inexpensive, killed antigen vaccine cannot be administered to birds owned by small scale producers and interested in protecting their flock provided there is a suitable veterinary professional or paraprofessional to perform the work and the owner is willing to pay for vaccination. This is being done already or is potentially available in both Vietnam and China - two countries that first introduced mass vaccination against Gs/GD/96-lineage H5Nx viruses. Issues that arise are those of application, including availability of a well-matched vaccine, providing sufficient doses at an appropriate time, a lack of interest/demand for vaccination from some very small scale, low input producers. Field observations and reports 
on mass vaccination in places with large numbers of smaller scale producers do suggest it is not always done well, based on levels of antibodies reported in places where these have been measured post-vaccination. Nevertheless, a community-based veterinary paraprofessional could provide this service to any household in much of SE Asia if the demand was there and vaccination was allowed (which it isn't in a number of countries where Gs/GD/96-lineage H5Nx viruses are endemic or where cases recur).

The term "small scale producers" is not defined. If this refers to household flocks of, say, 10 to 30 birds reared extensively then there are many logistical problems in delivering vaccine to these flocks. Many are multi-age, owners are not interested in vaccination and delivering vaccine to all birds at the appropriate age is difficult. One problem is that vaccine is produced for larger flocks in multidose bottles (see recommendations from OFFU meeting in Beijing). Another part of the problem is that the amount paid for administering vaccination in some places is too low to justify the expense of travel by veterinary paraprofessionals. However, in places where highly pathogenic avian influenza occurs seasonally and regularly, it would be possible to provide vaccination coverage to flocks in an area if required/demanded.

In places where outbreaks occur sporadically the signals and need for vaccination are weak.

Much of the infection with Gs/GD/96-lineage virus occurs in domestic ducks and for many strains the level of mortality produced is relatively low and age-dependent. There is little incentive for duck farmers to vaccinate unless they have experienced losses from this disease previously or have experienced losses due to government action when an outbreak occurs on nearby farms (such as whole village culls).

Even if a universal HPAI vaccine for poultry could be developed there is no guarantee it would be used by all producers, especially those rearing poultry in low input systems or for short-lived birds, especially if there is an outlet for birds in the event of an outbreak. Many farmers will sell birds when they see early signs of mortality and those with very small flocks are less likely to vaccinate than those with larger flocks (Delabouglise et al., 2020 ${ }^{1}$ ). In low input systems the loss of some birds is expected.

Vaccination is a cost to producers and in many countries rearing of broilers is a low margin business. This will result in some farmers choosing not to vaccinate even if effective vaccines are available.

The paper highlights the potential downsides of vaccination without necessarily balancing this with information on methods to overcome the concerns. For example, much is made of the antigenic variation restricting the effective life of vaccines. This has been the case in several countries in which the virus remains poorly controlled but not in others. Action has been taken to ensure appropriate vaccines are available in most countries albeit more slowly than required.

Several vaccines already offer broad cross protection against different clades within the Gs/GD/96-lineage.

The paper does not cover one of the biggest constraints to vaccination which is the right for farmers to access vaccines. In a number of countries, $\mathrm{H} 5$ avian influenza vaccine cannot be used legally despite the virus being endemic. 
There is also no mention of ring vaccination, as used in some countries (but discouraged in others) around outbreaks. There is also no discussion on the benefits of having immune birds delivered to live bird markets as has been seen with both H5Nx and H7N9 viruses reducing the likelihood of infection in birds and spill over to humans.

The following points on the authors text (in italics) should be considered if/when revising the paper. Note that some of the references cited were published after this paper was submitted but should still be considered. Papers cited on advances in vaccines are only examples.

Title:

The article is discussing vaccination against highly pathogenic avian influenza so this should be included in the tile.

\section{Extracts from the abstract :}

Vaccination against highly pathogenic avian influenza (HPAI) viruses, along with other measures, was successful in eradicating AI in very few countries where the competence of national veterinary services or the geography and bird density have contributed favorably to the outcome.

- The objective of vaccination against highly pathogenic avian influenza in most places was not elimination but, rather, to reduce levels of infection and the threat to humans. This decision was made because it was not possible to eliminate the virus using the methods generally applied, based on stamping out and movement management. Vaccination was used in Hong Kong and Mexico to help eliminate particular strains of highly pathogenic H5 avian influenza virus and in Hong Kong to supplement measures in place to prevent infection in markets.

Eradicate vs eliminate? WHO uses the former for global "elimination". I prefer use of elimination.

The main constraints to an effective AI vaccination are vaccine composition matching field strains, reliable cold chain, and logistics to target all poultry smallholders, constraints related to the availability of sufficient financial and human resources.

Matching vaccines to field strains is not as important as proposed here in all places where vaccine is being used (it is an issue that can be overcome, and methods are available for rapid production of matched vaccines). In southern Vietnam, the same vaccine remained suitable from 2005 for at least 5 years against clade 1 viruses until the dominant strain changed due to introduction of a new clade. Cold chains are important but not a constraint for delivering other poultry vaccines that require similar storage in SE Asia. Vaccination does not need to target all small-scale producers if the goal is disease containment. The cost of vaccination is only one of the issues. Other important constraints to use of vaccine are the life span of birds (e.g. very short life span for broilers in places such as Indonesia), interference from maternal antibody and as mentioned above not having the right to use vaccines due to national bans (e.g. not currently permitted in a number of Asian countries even in countries where the virus is endemic).

When not conducted properly, vaccination can also contribute to the emergence of new field viral 
strains, through genetic drifts of HPAI viruses.

Note that antigenic variation also occurs when vaccination is not used - e.g. H6 in China (Wang et al., 2014²). Antigenic change will occur slowly in places where vaccinated or immune birds are rarely exposed to virus. In Vietnam and Indonesia, the bigger driver of vaccination failure was the introduction of a novel strain of virus from outside the country. Antigenic drift was not a factor for Clade 1 viruses and vaccines in southern Vietnam over a number of years. Circulation of multiple strains of virus is also a constraint matching becomes more difficult when more than one antigenic variant is circulating.

Would use of vaccine be regarded as improper in places where infection is endemic and high-level population immunity across areas is hard to achieve but good immunity at the individual farm level is achievable? Much depends on the purpose of the vaccination programme.

While new technologies have improved the possibility to produce high quality vaccines matching field strains, recurrent issues like post-vaccination field surveillance and vaccination coverage continue to limit the relevance of AI vaccination in smallholder settings.

The extent of post-vaccination surveillance depends on the purpose of vaccination. In a country where the virus is endemic and there is no possibility of elimination in the short to medium terms ( 2 to 5 years or longer), expensive surveillance systems for all flocks cannot be justified.

A "gamechanger" vaccine targeting smallholders should be universal to protect against all field viral strains and reduce significantly, if not totally eliminate, the need for costly post-vaccination surveillance. The ease of administration of this vaccine (eye drop or one single injection) would further contribute to its relevance in the field. These characteristics are considered essential for the product profile of an AI vaccine that can contribute in a meaningful way to the livelihoods of poultry smallholders.

It would be good to have such a vaccine, but we can still vaccinate smallholder flocks even with existing products, provided care is taken to use the most appropriate vaccine. The main post-vaccination surveillance required is detection of antigenic variant strains. This is not expensive. Surveillance is only costly if the goal is virus elimination and detection of all infected flocks. This is not the case in places where viruses remain endemic. Surveillance programmes recommended for use in Europe are expensive and can be a barrier for use of vaccines.

\section{Main text:}

Although more than 30 epizootics of high pathogenicity Avian Influenza (HPAI) have been reported in poultry (Gallus domesticus) and other birds since 1959, vaccination of poultry has only been added as a control tool since 1995 (Swayne et al., 2014). In recent outbreaks, vaccination has been used only in about $19 \%$ of the countries experiencing HPAI 15 countries out of 80 countries), showing that vaccination is not the most common and immediate response to an outbreak (Swayne et al., 2011).

Vaccination is not (in most situations) the most common response. However, the reason for this low uptake is that in high income countries, if virus is detected early, stamping out is used successfully to eliminate the virus. There are some exceptions to this rule. Trade restrictions can also inhibit use of vaccines. There are also situations where vaccination could be used as an early response to an outbreak, but trade issues inhibit its use. 
The nature of the AI virus, with the rapid emergence of new field viral strains, through genetic drifts of HPAI viruses, has affected the effectiveness of vaccines.

In some countries there has been rapid emergence of antigenic variants within a few years, in other countries the vaccine strains used have been suitable for multiple years. However, most countries that are using vaccine have systems in place to update vaccine antigens.

Vaccination along with other measures has been successful in eradicating AI in very few countries where the competence of national veterinary services (e.g. France, the Netherlands) or the geography and bird density (Hong Kong) have favorably contributed to the outcome.

In which situation has vaccination been used to eliminate avian influenza in France or the Netherlands? They both used vaccination selectively as a preventive measure in 2006 but this was abandoned, in part, presumably because of the cost of the required surveillance. Hong Kong SAR farms were densely packed, and vaccination was used as a measure to help prevent infection not only in farms but downstream in live poultry markets. Vaccination was added to the measures to contain the outbreak in 2002 and 2003 but became a compulsory preventive measure in December 2003 for any bird (imported from mainland China or locally reared) destined to be sold in a live poultry market in Hong Kong SAR. No cases occurred in Hong Kong until 2008 when an antigenic variant strain affected one poultry farm and was detected in several markets. No cases have been recorded since. It was the structure of the industry in Hong Kong SAR that resulted in routine preventive vaccination (live birds sales, import of live birds from mainland China and biosecurity systems on farms that could not be improved sufficiently to be confident of preventing all viral incursions).

In the four countries (China, Egypt, Indonesia and Vietnam) where massive vaccination campaigns have been initiated since 2004 (accounting for more than 99\% of the use of AI vaccines), HPAI virus is now endemic, outlining the difficulty to eradicate AI in countries with a high percentage of backyard poultry (Swayne et al., 2011).

The virus was endemic before vaccination commenced in these countries and was used because virus elimination was not possible owing to the nature of the poultry production and marketing sector (Sims, 2016). Vaccination did not result in endemic infection - it was a response to it. Note also that certain strains of virus have disappeared (e.g. clade 7 viruses in China). Note also that vaccination has been used in Bangladesh for a number of years now. Vaccination has also been used illegally in a number of countries where it is banned. The presence of a high proportion of smallholder flocks is not necessarily an impediment to elimination (e.g. Thailand) even when vaccination is not used. Note that there is considerably more usage of H9N2 vaccination across Eurasia than against $\mathrm{H} 5 \mathrm{Nx}$ so when referring to AI vaccines ensure this states it is for HP viruses.

Orthomyxoviruses are classified as Types A, B or C with Avian influenza caused by a highly mutable Type A influenza virus.

And Type D. All type A viruses are highly mutable, not just avian influenza viruses

The antigenic variation of the HA and NA surface glycoproteins occurs at a high frequency through minor "drift" changes and may be associated with the immune pressure exerted by the vaccination of birds.

But there are other drivers apart from vaccination (see Wong et al., re H6). In some cases, vaccination has probably resulted in selection of an existing antigenic variant (e.g. H9N2 in Korea). 
Major antigenic "shift" in the HA and NA coding proteins, the result of genetic re-assortment between gene segments of two different influenza virus strains (subtypes) in host cells, commonly occurs especially when domestic waterfowl and poultry are in close proximity as often seen in developing countries under smallholder settings.

Does this reassortment occur in smallholder settings or in live bird markets? Also much of the recent reassortment has probably occurred in wild birds (King et al., 2020 ${ }^{3}$ ). There is a much greater likelihood of reassortment in environments where multiple strains of virus are circulating as was the case in markets in Hong Kong in 1997. Most of this reassortment involves internal protein genes. The $\mathrm{H} 5 \mathrm{~N} 1 \mathrm{combination}$ was favoured until the emergence of clade 2.3.4.4 viruses which have been shown to readily acquire different NA genes.

For Gs/GD/96-lineage viruses the HP H5 HA has not been replaced since 1996 so there has not been a true shift (involving a change in subtype). Drift has occurred and certain HA clades have emerged that are so antigenically dissimilar that vaccines are ineffective when a novel strain is introduced to a new area. For example, Indonesia with the introduction of Clade 2.3.2.1 viruses.

Ducks are often silent carriers of the AI virus, constituting an additional challenge for the control of AI (Swayne et al., 2013).

It is preferable to state that ducks can be infected silently given individual ducks rarely shed viable virus for a period beyond 10 days. They are not true carriers.

AI viruses in poultry are classified as either low pathogenic (LPAI) or highly pathogenic (HPAI), based on the clinical signs and mortality, using the definition established by the World Organization for Animal Health (OIE, 2018). During the period January 2013 to February 2019, HPAI in domestic poultry was reported in 68 countries and territories with a total of 7,270 outbreaks (OIE, 2019).

The number of outbreaks is in some ways misleading given most cases of infection are not reported/detected in countries where the virus is endemic. The majority of these HPAI outbreaks were caused by Gs/GD-96-lineage H5Nx viruses.

In most of the countries in Asia and Africa, the poultry sector is dominated by smallholder farmers.

- It would be good to define what you mean by smallholder farmers. It is much more difficult (but not impossible) to implement vaccination for small multi-age flocks of say $<50$ head than a commercial flock of say 500 birds. Note in Africa there is no vaccination against HPAI (officially), except in Egypt.

H5N1 HPAI viruses have been sub-classified according to Clades, or groups of AI viruses that share a common ancestor (WHO, 2011). There are at least 10 Clade groups currently identified by the OIE and FAO network of expertise on animal influenza (OFFLU).

This system applies to Gs/GD-96-lineage H5Nx viruses, not other H5 HPAI viruses. Although the initial classification divided the viruses into 10 clades 0 to 9, most of these clades have extinguished but viruses within Clade 2 have continued to evolve to form a number of $5^{\text {th }}$ order clades, especially within clades 2.3.2.1 and 2.3.4.4

Both antigenic shift and drift are important mechanisms for the evolution of the virus. The presence of co-circulating subtypes among dense populations of birds adds extra pressure for antigenic shift.

Delete - mentioned already but see earlier comments about shift and reassortment. 
Vaccination is also believed to exert a selection pressure on the virus by increasing the mutation rate by several orders of magnitude (Swayne et al., 2014).

This is probably true in places where there is a high likelihood of infection in vaccinated flocks (e.g. Egypt) but not in situations where there is a good match between circulating virus and the vaccine antigen or where incursions of virus are infrequent. Experimentally it is possible to select for antigenic variants using antiserum suggesting a role for antibodies, but we still do not know the precise contribution of vaccination vs natural infection, especially in ducks.

In addition, the evolution of new clade types can change the morbidity and mortality. Prior to 2012, clade 2.2 (Indonesia) and clade 1.1 (Cambodia) were predominant, with mortality in domestic ducks of less than 10\%. Following the introduction of clade 3.2.1....

Indonesia was clade 2.1 and derivatives. The introduced virus was clade 2.3.2.1c to both. Note that many healthy market-weight ducks have been found infected with clade $2.3 .2 .1 \mathrm{C}$ viruses based on results of market surveillance in Cambodia so infection is not uniformly fatal.

The type of poultry production is also critical to assess the risk of exposure. Birds kept for longer periods (laying hens, breeder birds and slow-growth meat birds) have a longer duration of potential exposure to AI virus than short-lived poultry (broiler-type meat birds).

Also important is the level of biosecurity in the premises in which birds are reared. Longlived breeder birds tend to be a lower risk because of the higher biosecurity measures in place. It is easier to obtain high level immunity in long lived meat birds than in white feathered broilers given the difficulty in giving 2 doses of vaccine during the short life span of the latter.

In addition to being considered as genetically modified organisms, the potential of live ND vectored vaccines to spread to non-target species and to unvaccinated flocks often raises concerns during the regulatory review and vaccine licensing process

Often?

Coverage of the ND vector vaccines seems disproportionate compared to other vaccines given they have not been widely deployed in most parts of the world.

There are several new experimental AI vaccine approaches not currently licensed for commercial use including wild type or attenuated LPAI; use of various vectors e.g. adenovirus, salmonella, avian leukosis and vaccinia; eukaryotic systems e.g. plants; and DNA vaccines, with a main objective being a universal vaccine covering against all subtypes of HPAI.

Other developments include improved adjuvants/immunostimulants (e.g. Toll-like receptor agonists, splicing to flagellar proteins etc.) and inclusion of M2e protein to broaden protection (see, for example, Li et al., 20204, Lin et al., 20205). You should also refer to duck virus enteritis vector vaccines because these potentially offer protection against another important viral disease that causes high mortality in ducks and hence give greater reason for vaccinating ducks (Chen et al., 20196).

I recommend splitting this section in two given most of these vaccines are not aiming to be universal. It would also be worth commenting more about mucosal immunity and cell 
mediated immunity - see Wang et al. $\left(2020^{7}\right)$.

Review of the vaccine database from The Center for Food Security and Public Health, Iowa State University showed that of 43 manufacturers listing vaccines for the control of Avian Influenza, 31 (72\%) are currently manufacturing vaccines combining AI with other antigens (CFSPH, 2018).

CFSPH is no longer providing this service and the link is broken. You can still point out that this was the situation several years ago. Across the paper much of the data are dated.

Irrespective of the vaccination schedule or the specific vaccines used, Tarigan et al. (2018) demonstrated that the birds were not protected throughout production. Without vaccination after 19 weeks, the birds failed to be protected after 38 weeks of age; with vaccination after 19 weeks, the birds failed to be protected around 58 weeks of age. The data clearly demonstrated showed that with laying or breeder birds the duration of immunity is one of the main limitation issues for life cycle protection in the field, especially in the smallholder setting.

The paper by Tarigan et al. was an important reminder that killed antigen vaccines may not give life-long immunity. However, a number of the farms using vaccine did not follow the recommendations of vaccine manufacturers in terms of dose schedules, providing some explanation for the poor results. One farm that did still had acceptable levels of antibody at 68 weeks.

Due to the antigenic drift of the AI virus in the field, there is a need to constantly update the composition of AI vaccines.

This is true for killed antigen vaccines and some vector vaccines, but some vector vaccines have shown good cross clade protection (e.g. HVT-AI).

Vaccination is expensive due to the need to conduct high quality post-vaccination surveillance to monitor the genetic and antigenic characteristics of circulating field viruses.

The cost of post-vaccination surveillance depends on the objectives of the surveillance program. If vaccinating in a country with endemic infection where the goal is to reduce the effects of the disease and levels of circulating virus then the main objective is to monitor for antigenic variant viruses. This is a small cost compared to the cost of a large-scale campaign.

Poor vaccination practices, insufficient vaccination coverage and lack of post-vaccination monitoring (as often seen in developing countries with large backyard poultry populations) can contribute to an endemic situation

This section of the document does not focus sufficiently on any of the potential positive aspects of vaccination. It is reasonable to suggest that vaccination of backyard poultry is difficult, but it also depends on the quality of services available to small scale producers. For example, in border areas in China adjacent to Vietnam all poultry are vaccinated on a regular basis to develop an "immune" buffer zone.

Table 2 - I am not sure of the need for Table 2. I would recommend rechecking the data for Asia and the Americas and whether these are deaths directly from HPAI or deaths plus deaths from culling. If the latter, then the number for the Americas is too low.

Table 3 does not contain multiple antigens introduced recently - now up to Re-11 and Re-12 
for H5 vaccines. Three H7N9 antigens have been introduced - the last one Re-3 only a few weeks ago

The most common bivalent or trivalent vaccine against $\mathrm{HPAI}$ is $\mathrm{H} 5 / \mathrm{H} 7$ vaccine used in China.

\section{Table 5:}

There has been consensus for a number of years that an improved vaccine specifically for ducks that also addresses another disease (DVE) would be preferable. One of the vaccines that does offer broad cross clade protection in chickens (HVT-AI) does not protect domestic ducks.

The OFFLU Beijing meeting concluded "Any country considering vaccination of household poultry should take into account the difficulties that have been encountered in implementing and sustaining these programs in some countries. Intensive programs have been implemented successfully for limited time period in some countries where public health was a priority." This should be included in the paper.

\section{On universal vaccines:}

The antigenic drift of the AI viruses in the field requires a constant surveillance usually poorly performed by national veterinary services in developing countries due to the lack of proper facilities, human and financial resources. In addition, the availability of universal vaccines would eliminate the need to regularly update vaccines, often affected by inefficient national regulatory review and approval processes for vaccines

This does not reflect the work that has been done in Vietnam, Indonesia, China and Egypt to monitor antigenic change and where new vaccine antigens have been introduced in response to antigenic drift or introduction of a new antigenic variant (e.g. clade 2.3.4.4b to Egypt). A universal vaccine might be feasible, but it is unlikely that this would be a single dose vaccine. HVT-AI vector vaccine meets some of these requirements.

Such a vaccine would be also available in countries considering adding vaccination to their AI control measures even though the current trend shows that fewer countries are willing to initiate AI vaccination. Vaccination stockpiles are now in place in several countries including the US and the Republic of Korea, indicating these could be used in the event of an H5Nx outbreak. These are both places that previously had not vaccinated against HPAI. Iran is also using vaccine against H5 avian influenza (Baradaran Seyed et al., 20198).

\section{Conclusion:}

Vaccination against HPAI has been used as one of the tools in national AI control programmes with varying degrees of successes

Measuring success depends on the objectives of the vaccination programme. In places like Vietnam the main reason for introducing vaccination was to reduce the number of human infections and to move away from culling millions of birds (many owned by smallholders) given this had not resulted in elimination of the virus. Similar for H7N9 vaccination in China. In none of the "endemic" countries was the goal elimination of virus.

Few countries have been able to eradicate HPAI and these countries have typically relied on extremely 
competent national veterinary services and / or had limited poultry population at risk.

Only true if you are referring to places that have used vaccination as part of an elimination programme.

The cost of AI vaccines is only a small part of the overall cost of vaccinating poultry against AI, especially when targeting poultry smallholders in lower-and middle-income countries. The current vaccine production technologies and vaccines have not been able to address the cost inherent to their use.

- Figures in your table 5 suggest otherwise in that the cost of administering the vaccine is lower than the cost of administration. See previous discussion on surveillance if the goal is not virus elimination.

In order to represent a significant advance, the profile for vaccine candidates must include protection against all viral strains (universal vaccine), efficacy in ducks with reduction in viral shedding and, protection after a single administration.

Universal vaccines tend be aimed at all strains of Influenza A virus. A duck specific virus that generates strong immunity would still be valuable. Single dose vaccination is already in use in ring vaccination around outbreaks.

Without the inclusion of these properties in the vaccine profile, it is unlikely that any vaccine candidate would add significant value to the current AI vaccine market and thus warrant the investment of funds for its development. In order to represent a significant advance, the profile for vaccine candidates must include protection against all viral strains (universal vaccine), efficacy in ducks with reduction in viral shedding and, protection after a single administration. Without the inclusion of these properties in the vaccine profile, it is unlikely that any vaccine candidate would add significant value to the current AI vaccine market and thus warrant the investment of funds for its development.

Existing vaccines can still provide protection against disease and reduce shedding. The logical approach to duck vaccination is to use a vector vaccine that also provides protection against another pathogen such as duck virus enteritis vaccine.

Poultry vaccines will not be developed specifically for the smallholder market. Many smallholder birds are not vaccinated for other important endemic diseases.

Some registered vaccines that afford good cross protection are available (see for example Oliveira Cavalcanti et al., $2017^{9}$ and Nassif et al., 202010). HVT vector can only be used in well-managed hatcheries.

\section{Additional references that could be included:}

1. Delabouglise et al. $\left(2020^{1}\right)$.

2. Wang et al. (2014²).

3. King et al. $\left(2020^{3}\right)$.

4. Li et al. (20204).

5. Lin et al. $\left(2020^{5}\right)$.

6. Chen et al. (20196). 
7. Wang et al. $\left(2020^{7}\right)$.

8. Baradaran Seyed et al. $\left(2019^{8}\right)$.

9. Oliveira Cavalcanti et al. (201799).

10. Nassif et al. $\left(2020^{10}\right)$.

\section{References}

1. Delabouglise A, Thanh N, Xuyen H, Nguyen-Van-Yen B, et al.: Poultry farmer response to disease outbreaks in smallholder farming systems in southern Vietnam. elife. 2020; 9. Publisher Full Text 2. Wang G, Deng G, Shi J, Luo W, et al.: H6 influenza viruses pose a potential threat to human health.J Virol. 2014; 88 (8): 3953-64 PubMed Abstract | Publisher Full Text

3. King J, Harder T, Conraths FJ, Beer M, et al.: The genetics of highly pathogenic avian influenza viruses of subtype H5 in Germany, 2006-2020.Transbound Emerg Dis. 2020. PubMed Abstract I Publisher Full Text

4. Li QY, Xu MM, Dong H, Zhao JH, et al.: Lactobacillus plantarum surface-displayed influenza antigens (NP-M2) with FliC flagellin stimulate generally protective immune responses against H9N2 influenza subtypes in chickens.Vet Microbiol. 2020; 249: 108834 PubMed Abstract | Publisher Full Text

5. Lin SY, Yao BY, Hu CJ, Chen HW: Induction of Robust Immune Responses by CpG-ODN-Loaded Hollow Polymeric Nanoparticles for Antiviral and Vaccine Applications in Chickens.Int J Nanomedicine. 2020; 15: 3303-3318 PubMed Abstract | Publisher Full Text 6 . Chen $P$, Ding $L$, Jiang $Y$, Zeng $X$, et al.: Protective efficacy in farmed ducks of a duck enteritis virus-vectored vaccine against H5N1, H5N6, and H5N8 avian influenza viruses. Vaccine. 2019; 37 (40): 5925-5929 Publisher Full Text

7. Wang T, Wei F, Liu J: Emerging Role of Mucosal Vaccine in Preventing Infection with Avian Influenza A Viruses. Viruses. 2020; 12 (8). Publisher Full Text

8. Baradaran Seyed Z, Pishraft Sabet L, Fallah Mehrabadi MH: Control of Highly Pathogenic Avian Influenza by Evidence-Based Vaccinology: Past Progress, Future Prospect. Iranian Journal of Epidemiology. 2019; 15 (1): 77-86 Reference Source

9. Oliveira Cavalcanti M, Vaughn E, Capua I, Cattoli G, et al.: A genetically engineered H5 protein expressed in insect cells confers protection against different clades of H5N1 highly pathogenic avian influenza viruses in chickens.Avian Pathol. 2017; 46 (2): 224-233 PubMed Abstract | Publisher Full Text

10. Nassif S, Zaki F, Mourad A, Fouad E, et al.: Herpesvirus of turkey-vectored avian influenza vaccine offers cross-protection against antigenically drifted $\mathrm{H} 5 \mathrm{Nx}$ highly pathogenic avian influenza virus strains.Avian Pathol. 2020. 1-10 PubMed Abstract | Publisher Full Text

\section{Is the rationale for the Open Letter provided in sufficient detail?}

Yes

Does the article adequately reference differing views and opinions? Partly

Are all factual statements correct, and are statements and arguments made adequately 


\section{supported by citations?}

Partly

\section{Is the Open Letter written in accessible language?}

Yes

\section{Where applicable, are recommendations and next steps explained clearly for others to} follow?

Partly

Competing Interests: No competing interests were disclosed.

Reviewer Expertise: I am an animal disease manager with over 25 years experience in the control and prevention of avian influenza, including use of vaccination.

\section{I confirm that I have read this submission and believe that I have an appropriate level of expertise to confirm that it is of an acceptable scientific standard, however I have significant reservations, as outlined above.}

Reviewer Report 21 October 2020

https://doi.org/10.21956/gatesopenres.14370.r29690

(C) 2020 Spackman E. This is an open access peer review report distributed under the terms of the Creative Commons Attribution License, which permits unrestricted use, distribution, and reproduction in any medium, provided the original work is properly cited.

\section{Erica Spackman}

Southeast Poultry Research Laboratory, United States Department of Agriculture (USDA)Agricultural Research Service (ARS), Athens, GA, USA

Although I generally agree with the conclusions, the authors seem to lean more "all-or-nothing" than I do when it comes to improvements in vaccines; incremental improvements would likely be beneficial. Also, if commercial poultry are being vaccinated in a region experiencing an AIV outbreak, including back yard poultry in vaccination efforts may be beneficial to food security, economic stability, and disease control (provision of resources by the government would probably be necessary in many situations). All ideal features listed for vaccines for backyard poultry would greatly benefit commercial poultry vaccination as well. Developing vaccines that fulfill these criteria will be difficult; capitalizing on any single improvement should be considered within the scope of a given situation. In general, this is a very good, succinct summary of the topic. A few comments on some statements from the letter are listed below add clarity and come additional context.

\section{General:}

How is "backyard/small holder" poultry defined in the letter? FAO sectors 3 and 4 may be appropriate. 


\section{Introduction:}

"...Indonesia and Vietnam) where massive vaccination campaigns have been initiated since 2004 (accounting for more than 99\% of the use of AI vaccines)..." - Note that HPAIV was probably already endemic when vaccination was initiated.

\section{AI - etiology and epidemiology:}

"...16 H strains and $9 \mathrm{~N}$ strains..." -The $\mathrm{H}$ and $\mathrm{N}$ refer to subtypes, not strains.

"AI viruses in poultry are classified as either low pathogenic (LPAI) or highly pathogenic (HPAI)..." - Importantly, this applies to gallinaceous poultry (the testing is done in chickens and ducks are considered poultry but as stated by the authors, they are often silent carriers even for strains that are deadly for chickens).

"H5N1 HPAI viruses have been sub-classified according to Clades, or..." - The clade system for the goose/Guangdong/1996 lineage refers to the H5 HA only, the NA subtypes and lineages vary.

\section{AI vaccines and vaccination:}

“...3) HA DNA vaccine adjuvanted;..." - Although licensed in the US this vaccine platform has not been utilized in the field (as of C. 2020).

Need context as to why the NDV vector is the focus. It's not widely used in the field because of interference from immunity to the vector as discussed. Of the vectored vaccines, HVT and Fowlpox are most common.

"...rHVTAIV has the advantage that it can be used in day-old chicks at the hatchery." - And can be also be administered in ovo.

"Vaccination with high-quality, registered vaccines, according to established protocols reduces resistance to infection..." - Should this be "increases" resistance? There is not much work on vaccination and infectious dose for AIV, what data are available (for inactivated vaccines) suggest that vaccination doesn't affect the infectious dose, which is consistent with how the vaccine induces immunity.

"Vaccination is expensive due to the need to conduct high quality post-vaccination surveillance to monitor the genetic and antigenic characteristics of circulating field viruses." - This is true of long-term vaccination, if the outbreak can be halted vaccine use may be stopped. However, surveillance may be conducted regardless of vaccination.

"As a rule, it is not recommended to vaccinate backyard poultry since maintaining adequate levels of immunity is extremely difficult." - Alternatively, if the birds have some immunity it might contribute to better food security and cost-benefit by improving survival and maintaining production if there is a high level of virus challenge in the field.

Table 5: "Mass application" - The only AIV vaccine which might be given by spray application are avian paramyxovirus type 1 (NDV) vaccine vectored products which is a very small proportion of vaccines that have been utilized (i.e. well below $4.5 \%$ ). 
Tables 5 and 6: "Use in multiple species" - When they are tested, the current inactivated vaccines usually work well in ducks. However, data on vaccines in ducks and some poultry species are lacking. Vectored vaccines are restricted to the hosts of the vector.

Is the rationale for the Open Letter provided in sufficient detail?

Yes

Does the article adequately reference differing views and opinions?

Yes

Are all factual statements correct, and are statements and arguments made adequately supported by citations?

Partly

Is the Open Letter written in accessible language?

Yes

Where applicable, are recommendations and next steps explained clearly for others to follow?

Not applicable

Competing Interests: No competing interests were disclosed.

Reviewer Expertise: My areas of expertise are avian influenza virus vaccines, diagnostics and pathobiology.

I confirm that I have read this submission and believe that I have an appropriate level of expertise to confirm that it is of an acceptable scientific standard.

Reviewer Report 21 October 2020

https://doi.org/10.21956/gatesopenres.14370.r29689

(C) 2020 Smith G. This is an open access peer review report distributed under the terms of the Creative Commons Attribution License, which permits unrestricted use, distribution, and reproduction in any medium, provided the original work is properly cited.

\section{Gavin J.D. Smith}

Programme in Emerging Infectious Diseases, Duke-NUS Medical School, Singapore, Singapore

Here the authors have provided a nice overview of the challenges in controlling highly pathogenic avian influenza, with a focus on the challenges and limitations of vaccination as a control measure. There is also a discussion of how vaccines and vaccination could be improved to help small holders, who are proportionately more impacted by HPAI outbreaks. Overall I find this an interesting discussion and a worthwhile contribution to the area, but there are some inaccuracies 
that should be addressed prior to indexing.

Primarily the authors are incorrect in assuming that control AI in poultry was the main driving force for the use of vaccines. Thailand did not vaccinate because they are a major poultry exporter and use of vaccines would jeopardise the ability to certify poultry was free of AI. So they used testing and culling. In Hong Kong there was a combination of measures used, with mass culling and testing regimes also implemented. Other countries used vaccination not just to reduce poultry death but to reduce overall levels of virus in poultry which leads to less human infection. It would therefore be helpful to have a more detailed discussion of the uses of vaccination in combination with other control measures.

And it is not just vaccination that drove viruses to become endemic in poultry. The presence of ducks, which are largely immune to HPAI and are frequently asymptomatically infected, is an important factor, particularly as vaccine are largely ineffective in them. There were also long delays in implementing any effective control measures in many countries, providing time for the virus to establish in poultry and frustrating control measures. Poor biosecurity can also contribute to endemicity.

Minor points:

1. Paragraph 2 (Para 2) there is also influenza D.

2. Para 3, enveloped or not is not relevant here. Also info on the number of HA and NA types is dated.

3. Is Table 1 correct, e.g. is the Oceanic H7N2 truly HPAI?

4. Para 8 starting with "The type of poultry production is also critical to assess the risk of exposure" needs some references.

Is the rationale for the Open Letter provided in sufficient detail?

Yes

Does the article adequately reference differing views and opinions?

Partly

Are all factual statements correct, and are statements and arguments made adequately supported by citations?

Partly

Is the Open Letter written in accessible language?

Yes

Where applicable, are recommendations and next steps explained clearly for others to follow?

Yes

Competing Interests: No competing interests were disclosed. 
Reviewer Expertise: Virus evolution, AI molecular epidemiology, vaccine strain selection.

I confirm that I have read this submission and believe that I have an appropriate level of expertise to confirm that it is of an acceptable scientific standard, however I have significant reservations, as outlined above.

Reviewer Report 12 October 2020

https://doi.org/10.21956/gatesopenres.14370.r29700

(c) 2020 Busani L. This is an open access peer review report distributed under the terms of the Creative Commons Attribution License, which permits unrestricted use, distribution, and reproduction in any medium, provided the original work is properly cited.

\section{Luca Busani}

Dipartimento di Malattie Infettive, Istituto Superiore di Sanità, Rome, Italy

In the manuscript "Are current avian influenza vaccines a solution for smallholder poultry farmers?" the authors present the situation of the Highly pathogenic avian influenza, a list of limiting factors for the vaccination in smallholder settings and their opinion on what the characteristics of the ideal vaccine to target the poultry smallholder segment should have.

The paper is presented as an overview, without any kind of analysis or original data presented.

The paper is well written and the items are clearly presented, but I would suggest to the authors to add some details about the focus of the manuscript, that is the smallholder poultry farms: in particular a clear definition (FAO presents some descriptions in the Chapter 1 "The socio-economic Importance of Family Poultry" http://www.fao.org/3/y5169e/y5169e02.htm\#TopOfPage) and descriptions (backyard flocks are often multispecies and multiage, with poor or no biosecurity measures, a complex setting for vaccination). This is in my opinion important, to give to the reader a clear picture of the challenges of vaccinating smallholder flock.

Moreover, the background presents information on avian influenza epidemics and vaccine schedules, but mainly for commercial poultry. Additional comments on the impact of the AI epidemics on smallholders and examples of vaccination in backyard flocks should be provided as comparison (Individual and flock immunity responses of naïve ducks on smallholder farms after vaccination with H5N1 Avian Influenza vaccine: a study in a province of the Mekong Delta, Vietnam (Huynh et al., 2019¹).

Among the positive key factors for the successful control of AI in poultry, the veterinary services capacity is mentioned, but I would also suggest to add the need of lab capacity for diagnosis of poultry diseases and the regular use of the lab by vet public services, whenever possible.

In table 6 , at the universal vaccine rationale a possible interpretation is if such universal vaccine would become available, also the surveillance could be avoided. I would suggest to clarify this sentence pointing out the need of basic surveillance to ensure detection of the circulating viruses 
and vaccination failures.

In table 6, "use in multiple species" I would also suggest to add "multiple ages".

\section{References}

1. Huynh HTT, Truong LT, Meeyam T, Le HT, et al.: Individual and flock immunity responses of naïve ducks on smallholder farms after vaccination with H5N1 Avian Influenza vaccine: a study in a province of the Mekong Delta, Vietnam.PeerJ. 2019; 7: e6268 PubMed Abstract | Publisher Full Text

Is the rationale for the Open Letter provided in sufficient detail?

Yes

Does the article adequately reference differing views and opinions?

Partly

Are all factual statements correct, and are statements and arguments made adequately supported by citations?

Partly

Is the Open Letter written in accessible language?

Yes

Where applicable, are recommendations and next steps explained clearly for others to follow?

Yes

Competing Interests: No competing interests were disclosed.

Reviewer Expertise: Public Health, infectious diseases, zoonoses

I confirm that I have read this submission and believe that I have an appropriate level of expertise to confirm that it is of an acceptable scientific standard, however I have significant reservations, as outlined above. 